\title{
An introduction to Stellar Evolution
}

\author{
Oscar Straniero ${ }^{1}$ \\ INAF-Osservatorio Astronomivo di Teramo \\ Via Maggini s.n.c., 64100 Teramo, Italy \\ E-mail: straniero@oa-teramo.inaf.it
}

Stellar evolution theory is among the greatest successes of modern astrophysics. It is a powerful tool to interpret many astronomical observations, such as the Colour-Magnitude diagrams of simple and complex stellar systems or the chemical composition of the stars. Stellar models are required to derive important parameters, like the age of various cosmic components or their distances. In addition, stellar models allow us to investigate the behaviour of matter and radiation in the extreme physical conditions taking place in stellar interiors.

In this lecture, I review the main ingredients needed to cook a stellar model. Few examples of the potential of stellar evolution in the interpretation of relevant astronomical observations are illustrated. A final section is devoted to core-He burning stellar models and the interplay between nuclear burning and convection that determines the evolution of many stars.

VI European Summer School on Experimental Nuclear Astrophysics Acrireale Italy

September 18-27, 2011

$1 \quad$ Speaker 


\section{Introduction}

With only few exceptions, astronomical observations provide us with direct information limited to the outermost layer of a star. This layer is called the photosphere. The rest of the internal structure is very opaque and, therefore, remains hidden to the sight of our telescopes. Helioseismology and, to a less extent, the detection of neutrinos produced by nuclear reactions, can be used to study the internal structure of the Sun, but the application of these techniques to other stars is still rather limited. As a matter of fact, our knowledge of stellar interiors relies on theoretical models, whose reliability depends on the accuracy of the physical inputs used to describe a stellar structure. Since the physical conditions experienced by matter and radiation within a star cannot be easily reproduced in laboratory, in most cases the reliability of the stellar physics cannot be directly tested by means of a standard (Galilean) experimental procedure. In some cases, however, it may be probed by comparing models predictions with particular astronomical measurements.

As it is well known, the main purpose of stellar evolution is to study the evolution of the physical and chemical properties of the stellar interiors. This goal is achieved by translating the physical conditions that are established inside a star and all the phenomena that govern the changes over time of these conditions in a set of differential equations. Solving this set of equations, with appropriate boundary conditions, provides the internal profiles of the various physical properties (temperature, pressure, density and the like) and chemical quantities (the abundances of the various species of ions, atoms and molecules). In this way, we may also derive the temporal evolution of various observable quantities, such as the stellar luminosity, the effective temperature (namely, the temperature at the base of the photosphere) as well as the surface chemical composition.

A reliable stellar evolutionary model is not only a powerful tool for understanding the internal structure of stars with different masses or belonging to different stellar populations. Among the many possible applications, stellar evolution results are used to model galaxies, to predict the rate of the various types of supernovae or to date stellar systems.. In addition, stellar models provide us with a natural laboratory to study fundamental physics and, in turn, to test new theories. As an example, the lower limit to the age of the Universe provided by the age of the oldest Galactic Globular Clusters was a clear indication of the need of the dark energy to explain the universal timescale, a fact known well before the recent discovery of the accelerated expansion obtained by means of high-redshift observations of type Ia supernovae.

This paper is a report of a lecture I gave at the VI European Summer School of Experimental Nuclear Astrophysics in Santa Tecla (Catania, Italy). In addition to this introduction and the final conclusions, the paper consists of 3 sections. In the next section, I review how to build up a minimal set of equations suitable to calculate a stellar evolution model. The third section illustrates how to use the results of the theoretical calculation to interpret various astronomical observations. Finally, in section 4 I illustrate the core-He burning phase, with particular attention to the questions related to the coupling between convection and nuclear burning, whose ultimate effect is the determination of the final $\mathrm{C} / \mathrm{O}$ ratio. 
This lecture has been designed for PHD students of fundamental physics, astronomy or astrophysics.

\section{A minimal set of stellar structure equations}

The following four differential equation describe the structure of a self-gravitating, spherically symmetric stellar model:

$$
\begin{aligned}
\frac{d P}{d M_{r}} & =-\frac{G M_{r}}{4 \pi r^{4}} \\
\frac{d r}{d M_{r}} & =\frac{1}{4 \pi r^{2} \rho} \\
\frac{d L}{d M_{r}} & =\varepsilon_{\text {grav }}+\varepsilon_{\text {nucl }}+\varepsilon_{v} \\
\frac{d T}{d M_{r}} & =-\nabla \frac{G M_{r} T}{4 \pi r^{4} P}
\end{aligned}
$$

They provide an appropriate description of the internal structure of the majority of the stars, where the hydrostatic equilibrium is well established and the effects of rotation and magnetic fields may be neglected. The first equation represents the equilibrium between the gravity field and the gas pressure. Here, $r$ is the radius (radial coordinate), $M_{r}$ is the mass contained within the sphere of radius $r$ (mass coordinate) and $P$ is the pressure at $r$. The second equation defines the continuity of the mass ( $\rho$ is the density). Then, the third is the energy conservation equation. The three epsilon coefficients represent the rate of energy generation or sink (per unit mass) as due to the release of gravitational energy $\left(\varepsilon_{\text {grav }}\right)$, nuclear energy $\left(\varepsilon_{\text {nucl }}\right)$ and neutrinos production $\left(\varepsilon_{v}\right)$. Finally, the last equation connects the temperature gradient to the energy transport (radiative, convective or conductive). The various energy transport mechanisms are implicitly included into the nabla quantity, where $\nabla=\frac{d \ln T}{d \ln P}$. There exists two extreme cases:

1) Fully radiative energy transport:

$$
\nabla=\nabla_{r a d}=\frac{3 \kappa r^{2} P}{4 a c G M_{r} T^{4}} F=\frac{3 \kappa r^{2} P}{4 a c G M_{r} T^{4}} \frac{L}{4 \pi r^{2}}
$$

2) Adiabatic convection:

$$
\nabla=\nabla_{a d}=\frac{\gamma-1}{\gamma \chi_{T}} \quad \gamma=\frac{C_{P}}{C_{V}} \quad \chi_{T}=\left(\frac{d \ln P}{d \ln T}\right)_{\rho, Y i}
$$

$C_{P}$ and $C_{V}$ are the specific heats at constant pressure and volume, respectively. The first case is appropriate for stellar layers where convection is absent and thermal radiation supply the whole 
heat flow. This condition is fulfilled, for example, in the envelope of massive main sequence stars. Here, $\kappa=\frac{1}{\rho \lambda}$ is the radiative opacity ( $\lambda$ being the photons mean free path) and $F=\frac{L}{4 \pi r^{2}}$ is the energy flow through the sphere of radius $r$. In case of convection, the actual temperature gradient is in the range $\nabla_{a d} \leq \nabla \leq \nabla_{r a d}$. The lower limit is representative of the conditions established in in the deep interiors, such as in the core of He-burning stars. In these cases the huge heat capacity of the material moved by the convective instability makes the convective energy transport very efficient compared to the radiative contribution. On the contrary, convection is no longer adiabatic in the external layer of a star, where the actual temperature gradient may approach the upper (radiative) limit. Finally, the contribution to the heat transport by conduction is usually negligible. One important exception is the high-density degenerate core that develops during the late phases of stellar evolution (RGB, AGB or White Dwarfs phases of stellar evolution). In that case, the highly degenerate electrons behave as an almost perfect gas $^{2}$, their mean free path being substantially larger than that of photons. In these conditions they drive a very efficient outgoing energy flow ${ }^{3}$. Formally, the same expression used for the fully radiative temperature gradient may be adopted also in the case of a conductive contribution to the energy flow; in that case $\kappa=\left(\frac{1}{\kappa_{\text {rad }}}+\frac{1}{\kappa_{\text {cond }}}\right)^{-1}$, where $\kappa_{\text {rad }}$ and $\kappa_{\text {cond }}$ represent the radiative and the conductive opacity, respectively ${ }^{4}$.

The solution of the 4 differential equations described above provides the 4 dependent variables $(r, L, P$, and $T)$ as a function of the mass coordinate $\left(M_{r}\right)$. A few additional quantities appears in these equations and should be properly calculated. In particular:

$$
\begin{aligned}
& \rho=f\left(P, T, Y_{i}\right) \\
& \kappa=g\left(\rho, T, Y_{i}\right) \\
& \varepsilon_{v}=h\left(\rho, T, Y_{i}\right) \\
& \varepsilon_{\text {grav }}=-T \frac{d S}{d t}=-\frac{d E}{d t}+\frac{P}{\rho^{2}} \frac{d \rho}{d t} \\
& \varepsilon_{\text {nucl }}=\sum_{k} Y_{i} Y_{j} \rho N_{A}\langle\sigma \mathrm{v}\rangle_{k} Q_{k}
\end{aligned}
$$

\footnotetext{
${ }^{2}$ Actually, in a Fermi gas, electrons staked (two by two) in the states of lowest energy cannot move in a state already occupied by other (two) electrons.

${ }^{3}$ because of this phenomenon, the cores of RGB or AGB stars are nearly isothermal.

${ }^{4}$ The conductive opacity is related to the thermal conductivity $\left(\zeta_{\text {cond }}\right)$ by: $\kappa_{\text {cond }}=\frac{4 a c T^{3}}{3 \rho \zeta_{\text {cond }}}$
} 
where: $Y_{i}=\frac{X_{i}}{A_{i}}$ is the specific abundance (fraction by number) of the chemical specie labeled i, $X_{i}$ and $A_{i}$ are the mass fraction and the atomic weight, respectively; $\mathrm{S}$ and $\mathrm{E}$ are the entropy and the internal energy (per unit mass), respectively; $N_{A}\langle\sigma \mathrm{v}\rangle_{k}$ is the usual astrophysical notation for a nuclear reaction rate (see, e.g. [1]); $Q_{k}$ represents the amount of nuclear energy released by the reaction $\mathrm{k}$. It is given by the difference of the atomic weights of reactants and products multiplied by $c^{2}$. In case of a nuclear process involving a neutrino emission, the corresponding energy must be subtracted by $Q_{k}$, due to the negligible neutrino cross section. The first of these equations (equation 7), which relates the density to the pressure, temperature and chemical composition, is the equation of state. It includes the contributions of all the particles (free electrons, nuclei, atoms, molecules....) plus radiation. In some cases, deviations from the classical perfect gas EOS, as due to quantum-relativistic effects as well as to particles interactions (such as electrostatic interactions), should be carefully considered (see [2]). The calculation of the opacity coefficient (equation 8) requires the knowledge of all the relevant processes of photon absorption and re-emission. Among them, the most important in the deep interiors is the scattering on free electrons (Thomson or Compton scattering). In the external layers, where the recombination of atoms and/or molecules occurs, photons absorption by bound-free or bound-bound transitions should be also taken into account. In general, these processes depend on the wavelength, so that the opacity coefficient should be intended as a mean value, the so called Rosseland mean opacity. The neutrino energy production rate (equation 9) should include several elementary processes, the most important of which are illustrated in figure 1. Since neutrinos are weak-interactive particles with an extremely small mass, once produced (in the central regions) they escape from stars without interact with the rest of the stellar matter. Thus, their production represents an energy sink.
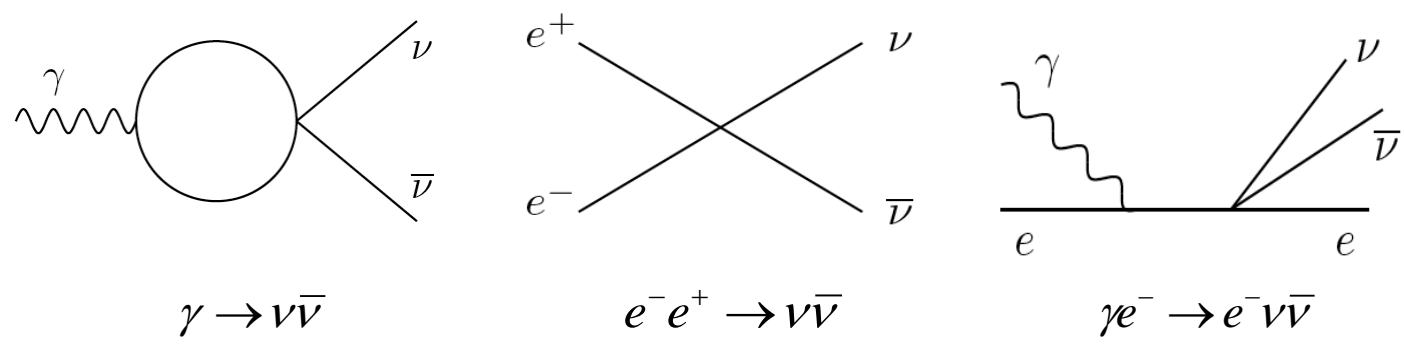

Figure 1.Thermal neutrinos processes. From left to right: plasma oscillation, pair annihilation, photo production (scattering on free electrons).

Plasma neutrinos production requires an high density electron gas, as it occurs in the degenerate core of red giant or AGB stars. Photo and pair neutrinos requires high temperature 
conditions. They are the dominant energy sink process in massive stars during the C-burning phase and beyond ${ }^{5}$.

The relation for the gravitational energy (equation 10) follows from the first principle of thermodynamics. In practice, the heat content changes because of an internal energy variation or of a compression/expansion of the gas. In both cases, an increase or a decrease of the heat content corresponds to an opposite variation of the gravitational energy. Finally, equation 11 provides the amount of nuclear energy released (per mass and time units). It is given by the summation of the products of the nuclear reaction rates (per unit mass) by the corresponding Q values. The summation should be extended to all the active nuclear reactions.

From the equations described so far, it is evident that the chemical composition plays a fundamental role. For this reason, in order to calculate the evolution of a star, we should also know how the composition changes under the combined action of nuclear reactions and mixing. The following equation may be used to account for such a variation of the chemical composition:

$$
\frac{d Y_{i}}{d t}=\left(\frac{d Y_{i}}{d t}\right)_{n u c}+\left(\frac{d Y_{i}}{d t}\right)_{m i x} \quad i=1, \ldots \ldots \ldots ., N
$$

The first time-derivative on the right-hand side represents chemical variations induced by nuclear reactions:

$$
\left(\frac{d Y_{i}}{d t}\right)_{n u c l}=\sum_{j} c_{j}^{i} \lambda_{j} Y_{j}+\sum_{j, k} c_{j, k}^{i} N_{A} \rho<\sigma \mathrm{v}>_{j, k} Y_{j} Y_{k}+\sum_{j, k, l} c_{j, k, l}^{i} N_{A} \rho^{2}<\sigma \mathrm{v}>_{j, k, l} Y_{j} Y_{k} Y_{l}
$$

where the first summation on the right hand side refers to weak interaction (such as $\beta$ decays or electron captures), while the other two correspond to 2 and 3 bodies nuclear fusions, respectively. A general expression for the second time-derivative in equation 12, which represents the variation of the chemical composition due to mixing processes, is:

$$
\left(\frac{d Y_{i}}{d t}\right)_{m i x}=D \frac{\partial^{2} Y_{i}}{\partial r^{2}}-\mathrm{v} \frac{\partial Y_{i}}{\partial r}
$$

The two terms on the right-hand side of this formula represent diffusive and advective mixing, respectively. $D$ is the diffusion coefficient while $\mathrm{v}$ is the average fluid velocity. In stellar interiors, mixing may be caused by various dynamical or secular instabilities. Among them, convection is the most frequent. When and where a given instability occurs is established by means of a stability criterion. For convection, the Ledoux criterion is often adopted (see, e.g., [3]):

\footnotetext{
${ }^{5}$ Normally, the main mechanism of stellar energy loss is the emission of electromagnetic radiation from the surface, but in during the late evolutionary phases of massive stars, it is largely exceeded by the emission of thermal neutrinos in the core (photo and/or pair neutrinos).
} 
$\nabla_{r a d}<\nabla_{a d}+\frac{\phi}{\delta} \nabla_{\mu}$

where:

$\phi=\left(\frac{\partial \ln \rho}{\partial \mu}\right)_{\mathrm{P}, \mathrm{T}} \quad$ and $\quad \delta=-\left(\frac{\partial \ln \rho}{\partial T}\right)_{P, \mu}$

Note that for a perfect gas $P=\frac{N a K}{\mu} \rho T$, so that $\phi=\delta=1$. If $\nabla_{\mu}=\frac{d \ln \mu}{d \ln P}=0$

(homogeneous layers), the Ledoux criterion reduces to the widely adopted Schwarzschild criterion $\nabla_{r a d}<\nabla_{a d}$. These two criteria follow from a very simple arguments: suppose that a certain volume of matter is moved up (or down) with respect the position where the hydrostatic equilibrium is ensured. If, after this displacement, it is more heavy than the environment (or more light), then buoyancy pushes it back to the starting position. It can be easily demonstrated that this condition occurs when the Ledoux stability criterion is fulfilled. In practice, when the Ledoux criterion is fulfilled, the radiative heat transport ensures the total flow of energy. On the contrary, where the radiative gradient exceeds the adiabatic gradient, the layer becomes dynamically unstable (convection). In this case, the total energy flow is given by $F=F_{\text {rad }}+F_{c o n v}$. The heat capacity of stellar matter is usually so large that the up and down mixing induced by convection ensures a very efficient mechanism of heat transport. Formula 5 shows that the radiative gradient linearly depends on the radiative opacity $(\kappa)$. In the cool external layers of low mass main sequence stars (like the Sun) as well as in red giant stars, due to the partial recombination of hydrogen atoms and molecules, the opacity attains quite large values. As a consequence, the radiative temperature gradient increases and, in turn, convective instabilities develop. On the other hand, the radiative gradient also depends on the total energy flux $\left(\frac{L}{4 \pi r^{2}}\right)$. As a results, a convective core may easily arise when an efficient nuclear burning takes place in the deep interior. This is the case, for example, of core-He burning stars. $\mathrm{H}$ burning main sequence stars may also develop a convective core, but they should be massive enough $\left(\mathrm{M}>1.2-1.3 \mathrm{M}_{\odot}\right)$, so that the temperatures are large enough for the activation of the $\mathrm{CNO}$ cycle. Note that low-mass stars burning $\mathrm{H}$ through the pp chain (like our Sun) have a radiative core.

Even if the minimal set of equations so far described may appear relatively simple, it implies the knowledge of several physical inputs whose understanding is the ultimate purpose of stellar evolution. All the 4 fundamental interactions (gravity, electromagnetism, weak and strong interactions) are simultaneously in action within a star. Understanding of stellar evolution means to take under control the fundamental physics The physical conditions vary of several order of magnitude from star to star. In the solar interior, for example, the temperature varies by more than 3 order of magnitude from the center to the surface. Today, the density in the solar core is about $100 \mathrm{~g} / \mathrm{cm}^{3}$. However, when the Sun will become a cool white dwarf, the density will be as large as $10^{8} \mathrm{~g} / \mathrm{cm}^{3}$, so that the stellar matter cannot remain in the gaseous state. When a white dwarf forms, the core is in a liquid state, but later on, when the temperature 
progressively decreases, a phase transition to the solid crystal phase occurs. Then, the equation of state suffers substantial changes during the evolution of a star. If the interior of a mainsequence star practically behaves as a classical perfect gas, quantum-relativistic effects should be carefully considered to describe the state of the electronic component in the core of a red giant. Coulomb interactions between ions and between ions and electrons affect the equation of state of the C-O core of an AGB stars as well as that of massive stars approaching the core collapse. Such a variety of environmental conditions influences the nuclear reaction rates. Actually in stellar interiors nuclei are screened by free electrons so that their effective charge is smaller than that of bare nuclei. However, to quantitatively evaluate the strength of this phenomenon, we should have a detailed description of the electrons state. Similarly, the equation of state is an essential prerequisite for reliable opacity calculation. In the next section I give some examples of the use of stellar models to understand how stars appear to an observer and how they change across the Universe.

\section{Stellar Evolution: a key to understand the macro-cosmos.}

In practice, photons emitted at the base of the photosphere are the unique sources of information available to us to study the physics of stars and their evolution. The basic information carried by these "particles of light" is commonly arranged in the so-called colormagnitude diagram (CM diagram, also Hertzsprung-Russell diagram). The CM diagram of nearby stars (namely, within 100 parsecs from the Sun), whose parallaxes have been measured by the Hipparcos satellite, is shown in figure 2 . We may clearly distinguish an extended main sequence, the red giant branch and a clump of core-He- burning stars. The evolutionary tracks of two models with 1 and $5 \mathrm{M}_{\odot}$, respectively, are reported in figure 3. This is the theoretical counterpart of the CM diagram of figure 2 .

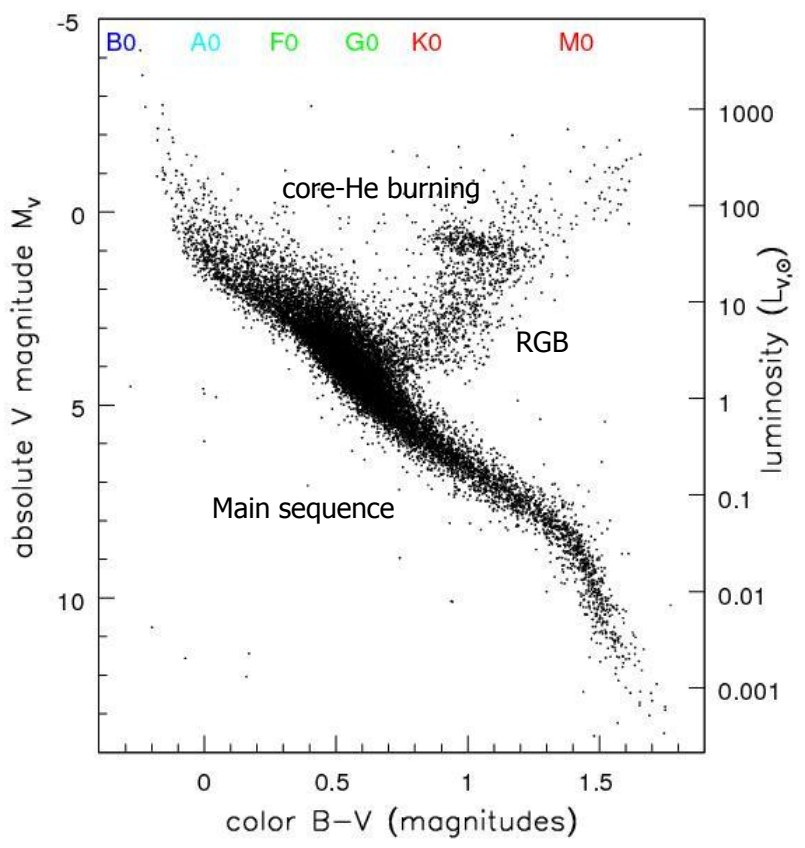


Figure 2.The Hertzsprung-Russell diagram of stars within 100 parsec whose distances were derived from parallaxes measurements performed by the Hipparcos satellite.

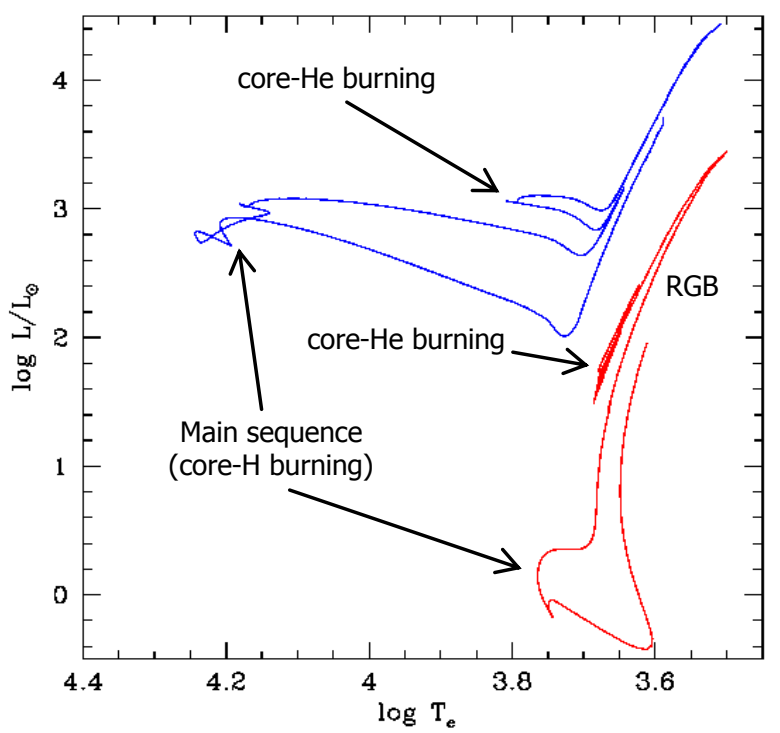

Figure 3. Tracks in the L-Te diagram of two evolutionary sequences corresponding to models with initial mass 1 and $5 \mathrm{M}_{\odot}$, respectively. Each evolutionary track illustrates the temporal variation of the luminosity and the effective temperature of a stellar model. The tracks here reported starts from the first model in hydrostatic equilibrium (on the so called Hayashy track) and follows its evolution trough the main nuclear burning stages ( $\mathrm{H}$ and $\mathrm{He}$ burnings)

The reproduction of these diagrams is one of the most striking successes of the stellar evolution theory. By comparing the theory with these observations, we can derive important parameters characterizing the cosmic environment. As an example, the CM diagrams of stellar clusters allow us to trace the history of the Galaxy. We can obtain the star formation history or the mass function ${ }^{6}$ at different epochs. We can also derive the age of the various galactic components (halo, disk and bulge) as well as a lower limit to the age of the Milky Way and, in turn, of the Universe. The CM diagram of M3, a typical Globular Cluster belonging to the galactic halo, is reported in figure 4. Each point represents a star of the cluster; overimposed, is a line representing a 13 Gyr theoretical isochrones?

${ }^{6}$ The (initial) mass function is the number of stars with masses in the range $M$ to $M+d M$ : IMF $=N(M) d M$. It has been shown that a power low function $\left(\operatorname{cost} \cdot M^{-\alpha}\right.$, where $\left.\alpha=2.35\right)$ provides a good reproduction of the IMF in the solar neighborhood (Salpeter IMF, [4]).

${ }^{7}$ An isochrones represents the loci of the points with equals age and chemical composition, but different mass. The faintest point in the $13 \mathrm{Gyr}$ isochrones shown in figure 4 corresponds to the position of a model with $\mathrm{M}=0.5 \mathrm{M}_{\odot}$, while the brightest point corresponds to a model of about $0.85 \mathrm{M}_{\odot}$. 


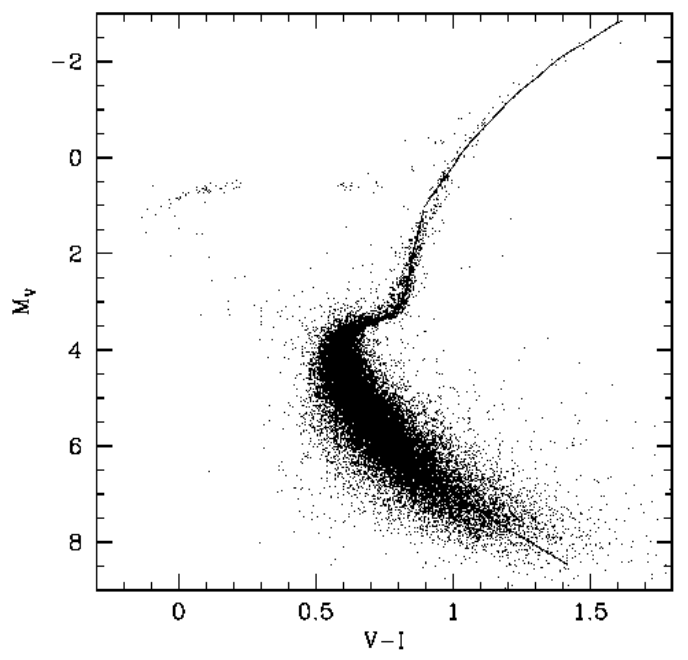

Figure 4. CM diagram of the Globular Cluster M3. The line is a theoretical isochrones of $13 \mathrm{Gyr}$ (from [5])
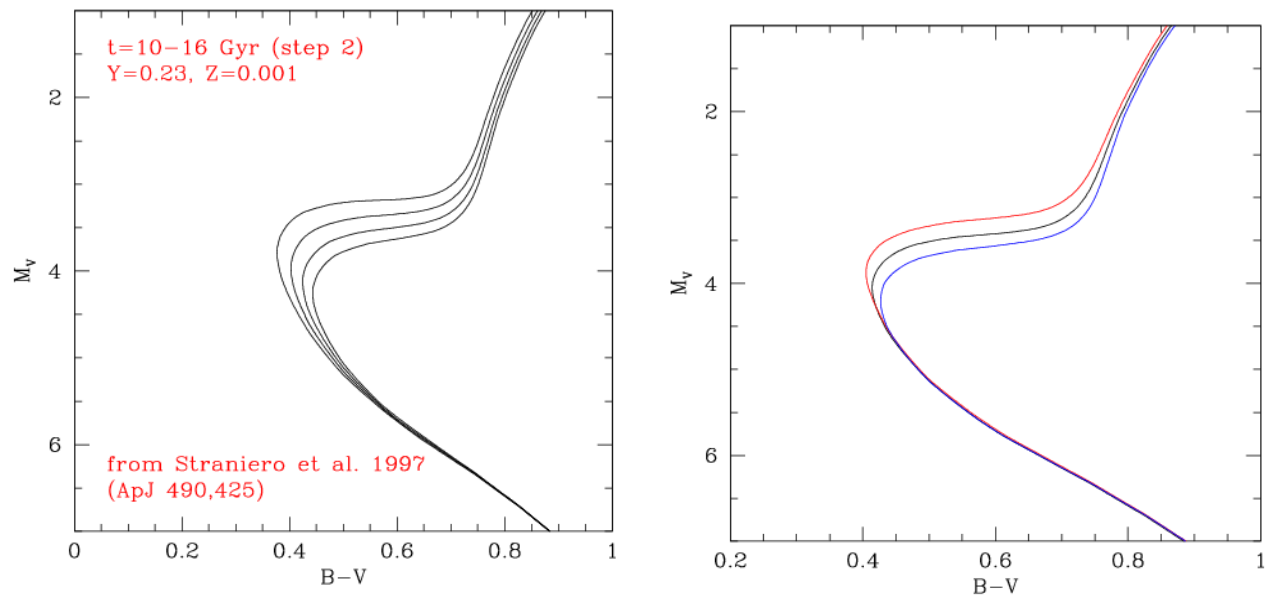

Figure 5. The age of a GC can be derived by measuring the luminosity of the turn-off (i.e. the upper limit of the main sequence): the brighter the turn-off, the younger the Cluster. This occurrence is illustrated in the left panel, where 4 isochrones with 10,12,14 and 16 Gyr are reported. In the right panel, 3 isochrones with the same age (14 Gyr), but computed by varying the rate of the ${ }^{14} \mathrm{~N}(\mathrm{p}, \gamma)^{15} \mathrm{O}$ reaction, are shown. This example clearly illustrates how an erroneous evaluation of the reaction rate leads to a systematic error in the estimated GC age (see [6]).

The precision with which we can evaluate these astronomical parameters certainly depends on the quality of the astronomical images and on the measurements of some cluster properties, such as the chemical composition, the distance and the light extinction along the line of sigh. Nevertheless, the reliability of the theoretical models is an essential requirement. Figure 5 illustrates the importance of the input physics adopted in the computation of stellar model on the derivation of the age of a Globular Cluster.

A more realistic comparison between theory and observations can be obtained with a synthetic CM diagram (figure 6). In this case, a Monte Carlo method is used to populate the $\mathrm{CM}$ diagram with the points representing the positions occupied by (synthetic) stars with 
different masses, but same age. Values of the stellar mass are extracted according to the assumed mass function. Then, from the theoretical evolutionary tracks we derive the pairs $(\mathrm{L}, \mathrm{Te})$, eventually translated into the observational diagram by means of appropriate luminositymagnitude and effective temperature-color transformations. We may also consider the effects produced by the statistical errors of the astronomical measurements. For this purpose, a relation between the photometric error and the luminosity is usually provided by the authors of the photometry. A certain fraction of unresolved binaries may be eventually considered.

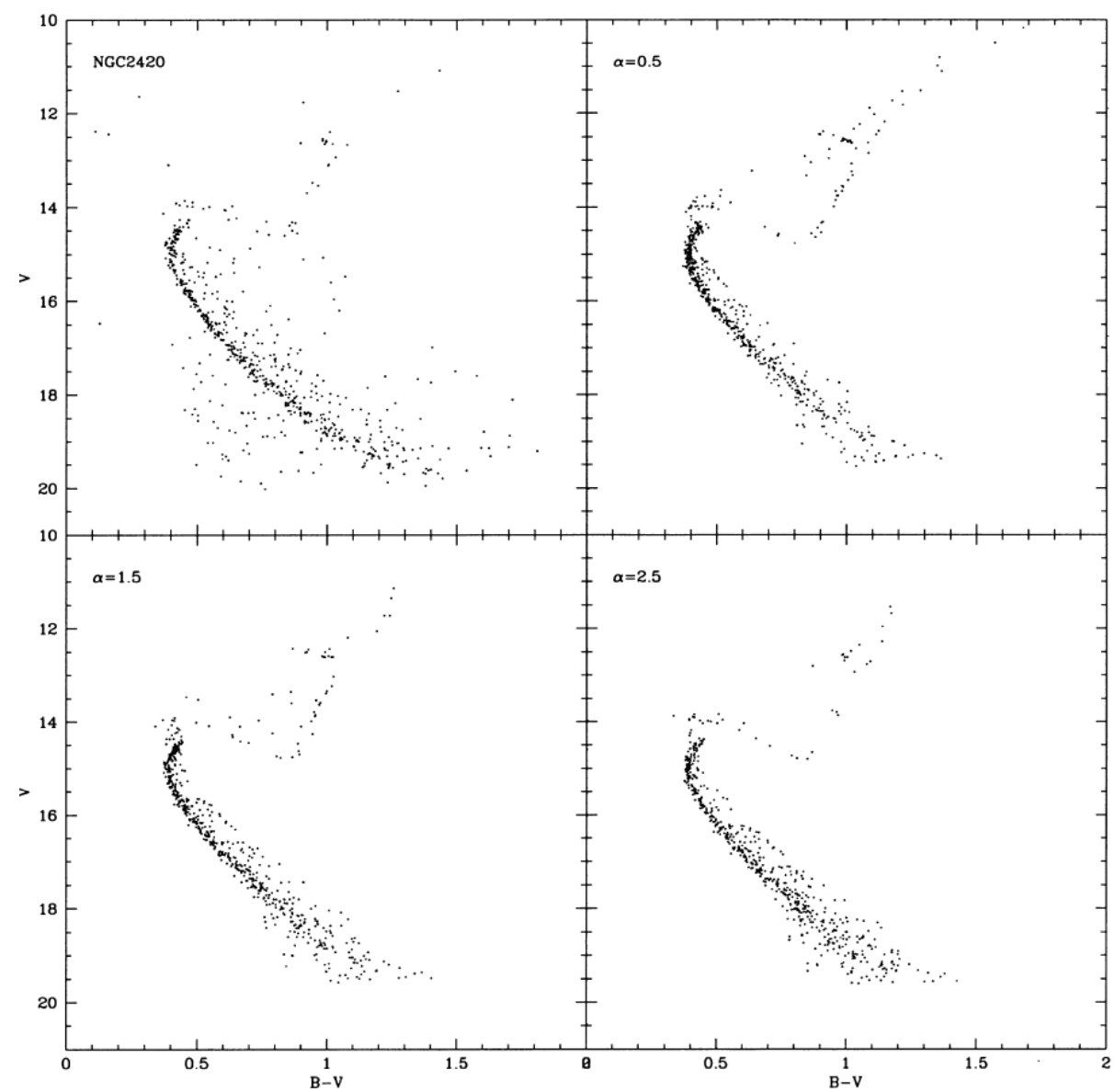

Figure 6. left-top panel: CM diagram of the open cluster NGC 2420. Top-right and bottom panels: synthetic $\mathrm{CM}$ diagrams as computed by assuming the same age $(1.5 \mathrm{Gyr})$, the same photometric errors, the same fraction of unresolved binaries (30\%), but different exponent of the power low IMF, namely $\alpha=0.5,1.5$ and 2 , respectively.

The CM diagram of NGC2420, an open cluster in Gemini, is shown in the top-left panel of figure 6 (adapted from [7]), A few example of synthetic diagrams are shown in the other 3 panels. Photometric errors and $30 \%$ of unresolved binaries have been considered. The 3 synthetic diagrams differ for the value of the exponent of the power low mass function.

Stellar nucleosynthesis is a further example of the power of stellar evolution theory as a tool for the interpretation of the cosmic evolution. Only $\mathrm{H}$ and $\mathrm{He}$, plus a very small amount of light elements ( $\mathrm{D}, \mathrm{Li}$ and $\mathrm{Be}$ ), were produced in the early Universe, 3 to 20 minutes after the Big Bang (more precisely, after the beginning of the space expansion). As a matter of fact, the 
chemical diversity we observe in the present-day Universe is the result of the nucleosynthesys occurred in stellar interiors, since the first stellar generation formed after the reionization epoch. Our understanding stellar nucleosynthesis relies on the availability of reliable stellar models. In addition to the reaction rates, the key ingredient for a reliable estimation of the stellar yields are the algorithms used to describe the mixings, those induced by convection and those eventually induced by other source of dynamical or secular instabilities. Mixing in stellar interiors has a twofold function. First of all, it allows the supply of fresh fuel in the higher temperature zones, where the nuclear reactions are active. On the other hand, the mixing provides an efficient mechanism to moves the ashes of the nuclear burnings toward the stellar surface. When this occurs, the interstellar medium may be polluted by the stellar wind made of material enriched with the products of the internal nucleosynthesis. In such a way, stellar evolution drives the chemical evolution of the cosmic baryonic matter

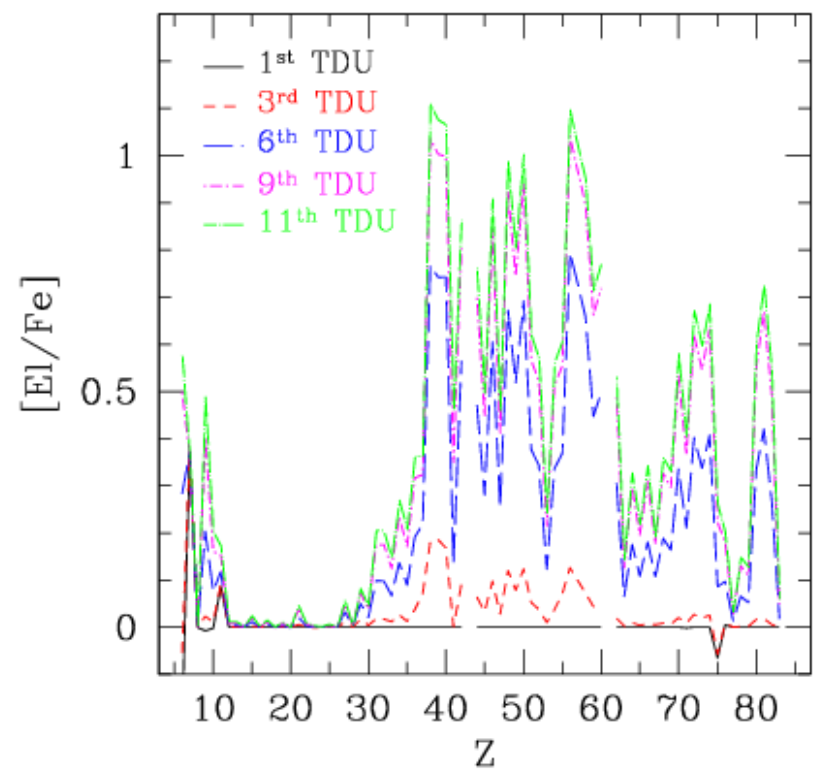

Figure 7. Composition changes of a $2 \mathrm{M}_{\odot}$ stellar model during the AGB phase. According to the legenda, each line represents the surface chemical composition after a certain number of third dredge-up (TDU) episodes. This figure is the result of the coupling between the complex network of nuclear reactions at work in the He-rich mantel and the recurrent penetration of the convective envelope into the $\mathrm{H}$-exhausted zone. Note that the initial composition coincides with the solar composition (i.e. $[\mathrm{El} / \mathrm{Fe}]=0$ ).

. Such a coupling between nuclear burning and mixing is at work in AGB stars. AGB stars undergoing recurrent thermal pulses and third dredge-up episodes are among the major polluters of the interstellar medium. The evolution of the chemical composition at the surface of a thermal pulsing AGB star $\left(M=2 M_{\odot}\right)$ is reported in figure 7 . In order to obtain this figure, Cristallo et al. [8] have computed about $2 \times 10^{5}$ stellar models ( $2 \times 10^{5}$ time steps). Each stellar model is made of 1000 to 5000 mesh points (this number depends on the complexity of the physical structure). For each mesh point, a set of 4 differential equations for the physical variables (see equations 1 to 4 ) and about 500 differential equations for the chemical species (see equation 12) has been simultaneously solved. 


\section{Core-He burning stars}

Core He burning stars are a good example to illustrate the importance of the coupling between nuclear burning and mixing and its influence on the stellar evolution.

In a low mass star $\left(\mathrm{M}<1.8 \mathrm{M}_{\odot}\right)$ the He ignition is preceded by the RGB phase, during which a He core grows in mass as a consequence of the shell-H burning. The approach to the He burning is influenced by two physical phenomena. The first one is the cooling induced by the (plasma) neutrino, whose production is more efficient near the center of the star where the density is larger. This occurrence causes a displacement of the maximum temperature, from the center to a point approximately located at $0.2 \mathrm{M}_{\odot}$ from the center. The He ignition occurs when the He-core mass attains a critical value of about $0.5 \mathrm{M}_{\odot}$ (the precise value actually depends on the chemical composition). Note that the $3 \alpha$ reactions start near to the maximum temperature point. The second important phenomenon concerns the equation of state in the core, which is dominated by the pressure of degenerate electrons. In non-degenerate conditions, the onset of a thermonuclear burning produce an expansion of the stellar structure that counterbalance the increase of the temperature caused by the nuclear energy release. In the core of an RGB star, however, the pressure of the degenerate Fermi gas depends only on density. Then, when the He burning starts, the increase of the local temperature is not balanced by a corresponding increase of the pressure. Nevertheless, due to the steep relationship between reaction rates and temperature, a positive feedback is established, resulting in a thermonuclear runaway. This is also called the He flash. It ends when the temperature becomes large enough to remove the electron degeneracy. Later on, the condition for the He burning are attained in a more central zone and a new flash stars. Actually, there will be a series of flashes, each one weaker than the previous one and closer to the center, until the electron degeneracy is removed in the whole core and a central quiescent $\mathrm{He}$ burning settles on (see figure 8). More massive stars does not develop a degenerate core, so that the He burning is quiescent (and centrally located) since the beginning. Except for the ignition, the He-burning proceeds in a similar way in all stars, irrespective of their mass. While the $3 \alpha$ reactions convert ${ }^{4} \mathrm{He}$ into ${ }^{12} \mathrm{C}$, a quite extended convective core settles on. As discussed in section 2, the development of the convective instability is the natural consequence of the huge energy flux generated in the central region of the star. 


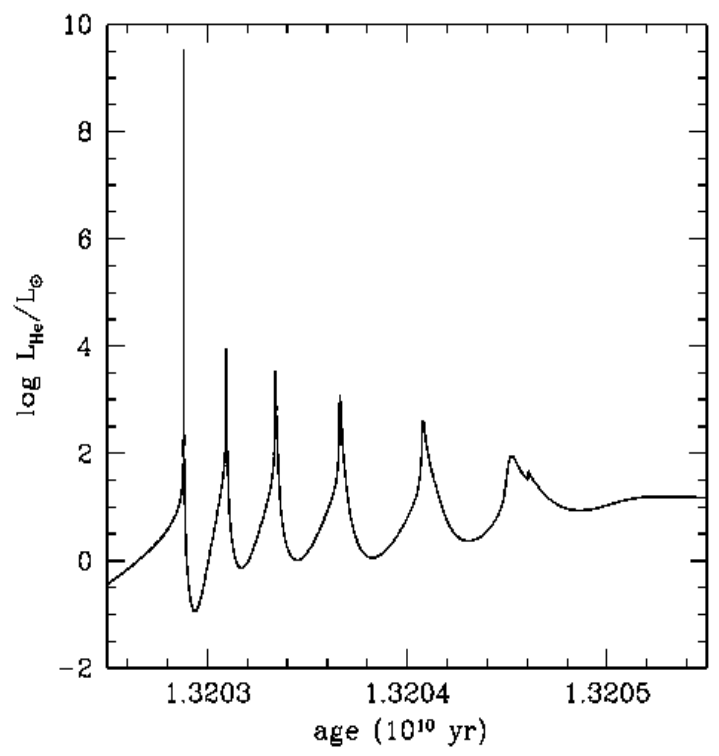

Figure 8. The series of He flashes occurring close to the RGB tip in a star of $1 \mathrm{M}_{\odot}$ and solar composition. The first (the strongest) thermonuclear runaway almost attains $10^{10}$ solar luminosities.

Later on, when a sufficient amount of carbon is accumulated, the ${ }^{12} \mathrm{C}(\alpha, \gamma){ }^{16} \mathrm{O}$ reaction enters in competition with the $3 \alpha$ and some oxygen is produced (see figure 9). As helium is progressively converted into carbon and oxygen, the opacity of the He-C-O mixture increases, the radiative gradient increases and, in turn, the mass of the convective core increases. Thanks to the large fuel reservoir provided by this growing convective core, the nuclear burning can last about hundred million years, replacing most of the energy lost from the surface as electromagnetic waves. A not negligible contribution to the energy budget also comes from the H-burning shell located at the base of the H-rich envelope, just outside the He core.

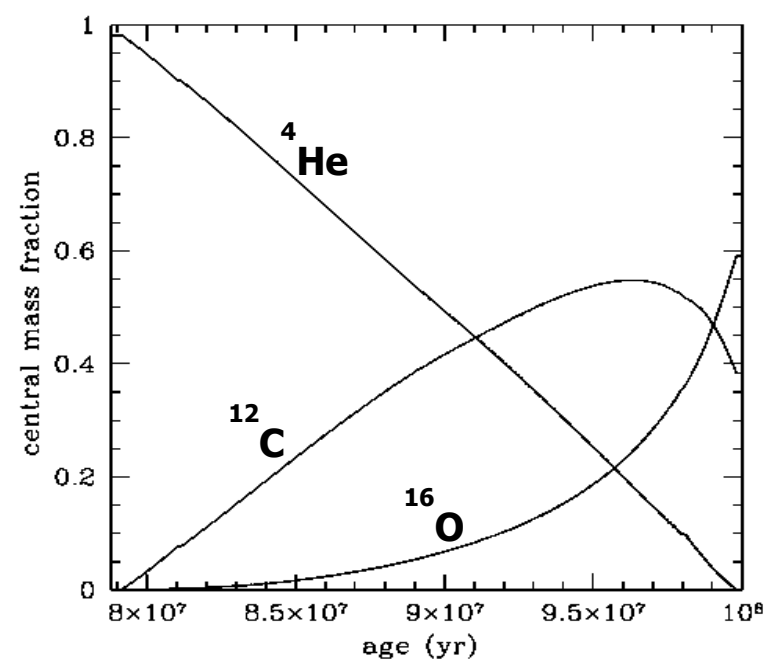

Figure 9. The evolution of the central abundance of $\mathrm{He}, \mathrm{C}$ and $\mathrm{O}$ during the core He burning of a $5 \mathrm{M}_{\odot}$ model.

The final $\mathrm{C} / \mathrm{O}$ ratio left by the He burning is a fundamental quantities affecting the late evolution of stars of any mass. Low and intermediate mass single stars are the progenitors of 
$\mathrm{CO}$ white dwarfs. The cooling timescale of these compact objects substantially depends on the $\mathrm{C} / \mathrm{O}$ ratio (see [9]). For example, the transition between the liquid and the solid phase occurs faster for oxygen than for carbon, due to the larger electrostatic potential. When these stars belong to close binary systems, they may become progenitors of type Ia supernovae. As shown by [10], the amount of ${ }^{56} \mathrm{Ni}$ produced by the explosion and, in turn, the light curve features, depends on the $\mathrm{C} / \mathrm{O}$ ration in the core of the WD progenitor. Straniero et al. [11] studied the influence of the uncertainties of the convective mixing efficiency and extension, as well as of the uncertainty of the ${ }^{12} \mathrm{C}(\alpha, \gamma){ }^{16} \mathrm{O}$ reaction rate on the $\mathrm{C} / \mathrm{O}$ ratio left by the He burning. Some example are shown in figure 10.

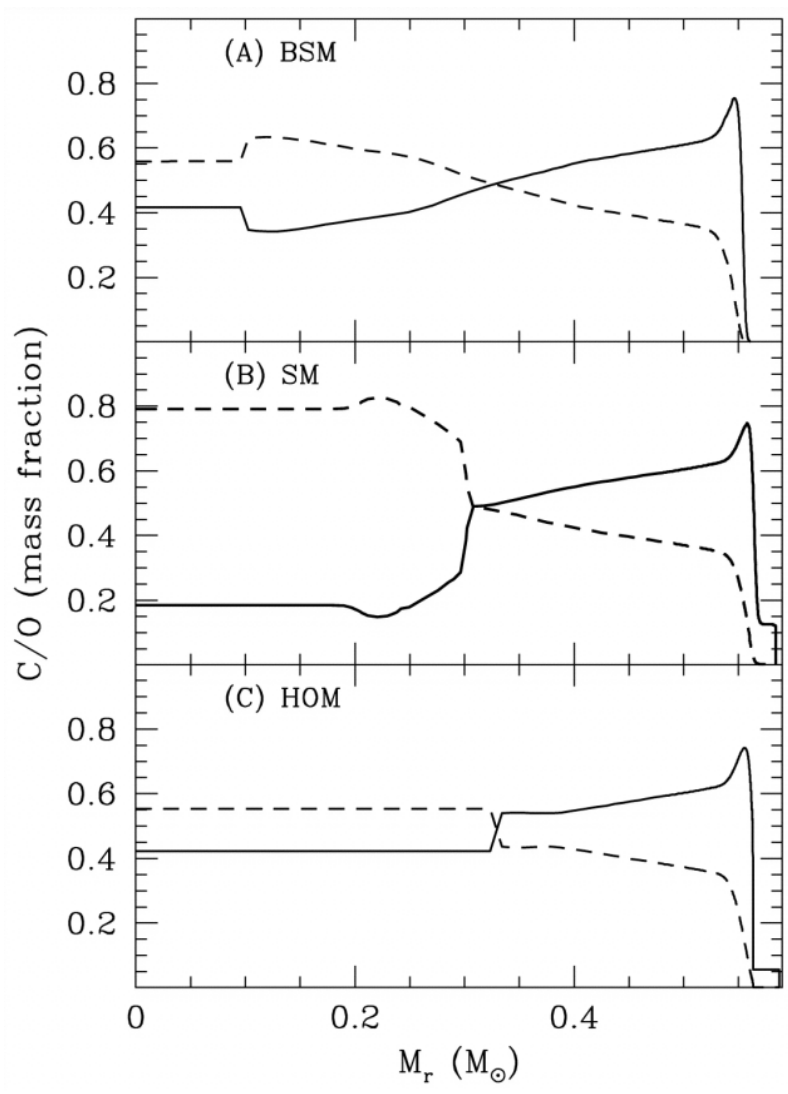

Figure 10. Chemical stratification within the core of a $2 \mathrm{M}_{\odot}$ model at the beginning of the thermal pulse AGB phase: $\mathrm{C}$ (solid line) and $\mathrm{O}$ (dashed line). Panel A) report the case of a models obtained by adopting a Bare Schwarzschild criterion, no overshoot and no semiconvection; panel B) the case of semiconvection; panel C) the case of mechanical overshoot $\left(1 H_{p}\right)$. Note the variation of the extension of the central, chemically homogeneous region that is the reminiscence of the convective core (adapted from [11]).

Semiconvection natuarally arises in a zone just outside the fully convective core of an $\mathrm{He}$ burning star. In this zone, which is initially dynamically unstable, the ingestion of fresh He from outside causes a decrease of the opacity and, in turn, a decrease of the radiative gradient, so that a negative feedback settle on that limit further mixing. As a result, mixing regulates itself in such a way that in the whole semiconvective layer the marginal stability condition, namely $\nabla_{r a d}=\nabla_{a d}$, is established. However, if a substantial mechanical overshoot occurs at the 
external border of the fully-convective core, the semiconvective layer is engulfed and, then, canceled. The evolution of the internal He profiles in the cores of semiconvective and overhooting models are compared in figure 11.
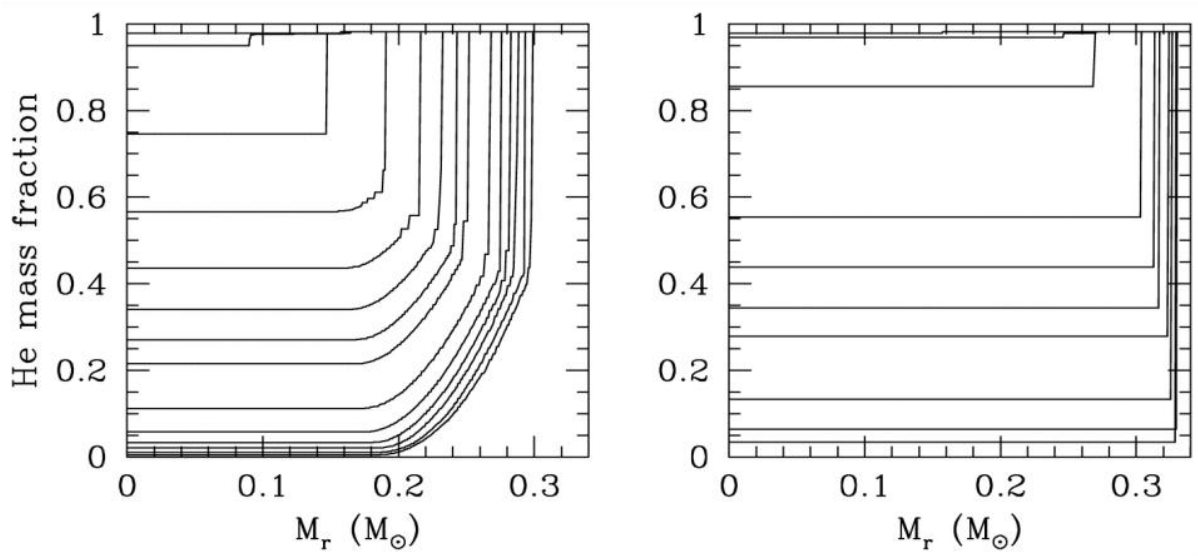

Figure 11. Evolution of the internal He profile for the semiconvective model (left panel) and for the $1 H_{p}$ overshoot model (right panel). Note, in the left panel, the development of the (flat) central fully-convective core and, just outside, the semiconvective layer. In the right panel, the semiconvective layer has been completely erased by the action of the convective-core overshoot [adapted from [11]).

Finally, the quantitative influence of the combined variations of the adopted convective scheme and of the ${ }^{12} \mathrm{C}(\alpha, \gamma){ }^{16} \mathrm{O}$ reaction rate is summarized in the next table:

Core He-burning Models: $M=3 M_{\odot}, Z=0.02$

\begin{tabular}{|c|c|c|c|c|c|}
\hline $\begin{array}{l}\text { Label } \\
\text { (1) }\end{array}$ & $\begin{array}{l}f^{\mathrm{a}} \\
(2)\end{array}$ & $\begin{array}{c}\tau_{\mathrm{He}}^{\mathrm{b}} \\
\text { (3) }\end{array}$ & $\begin{array}{l}X_{\mathrm{C}}{ }^{\mathrm{c}} \\
(4)\end{array}$ & $\begin{array}{l}X_{\mathrm{O}}{ }^{\mathrm{c}} \\
(5)\end{array}$ & $\begin{array}{c}M_{D}{ }^{\mathrm{d}} \\
(6)\end{array}$ \\
\hline 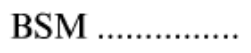 & 1 & 88 & 0.42 & 0.56 & \\
\hline 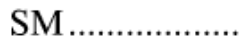 & 1 & 145 & 0.19 & 0.79 & 0.31 \\
\hline PSM....................... & 1 & 134 & 0.40 & 0.58 & 0.27 \\
\hline 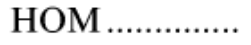 & 1 & 153 & 0.42 & 0.56 & 0.32 \\
\hline LOM................... & 1 & 139 & 0.38 & 0.60 & 0.28 \\
\hline SM & 0.4 & 135 & 0.52 & 0.46 & 0.29 \\
\hline SM & 1.6 & 149 & 0.08 & 0.90 & 0.31 \\
\hline HOM ...................... & 0.4 & 142 & 0.66 & 0.32 & 0.31 \\
\hline 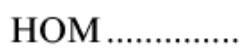 & 1.6 & 157 & 0.28 & 0.70 & 0.32 \\
\hline
\end{tabular}

a. Enhancement factor of the ${ }^{12} \mathrm{C}(\alpha, \gamma){ }^{16} \mathrm{O}$ rate. $f=1$ corresponds to the Kunz et al. (2002) rate.

b. He-burning lifetime (Myr).

c. Final central mass fractions of $\mathrm{C}$ and $\mathrm{O}$.

d. Location, in $\mathrm{M}_{\odot}$, of the sharp discontinuity that marks the separation between the innermost low-C zone, corresponding to the maximum extension of the convective core, and the external layer built up by the shell-He burning occurred during the AGB phase (see figure 10).

Concerning massive stars, the progenitors of core collapse supernovae, the influence of the $\mathrm{C} / \mathrm{O}$ ratio left by the He burning on the final explosive outcome has been discussed by several 
authors (see, e.g., [12], [13]). In summary, a lower amount of $\mathrm{C}$ makes the C-burning less efficient and, as a consequence, leads to pre-collapse stars with smaller iron-core mass. Note that a smaller core mass implies a more powerful explosion. In fact, as a consequence of the core collapse, a hard neutronised region forms in the innermost part of the iron core. Outside, the still falling material bounces on the surface of the newly formed hard inner core, where a shock wave starts to move outward. In order to emerge from the core, giving rise to the supernova explosion, the shock should travel through the still falling iron core material. In doing that, it loses energy by photodisintegration: about $10^{51}$ erg to cross a layer $0.1 \mathrm{M}_{\odot}$ thick. For this reason, when the shock reaches the base of the envelope, the residual energy will be larger if the presupernova iron-core mass is smaller.

Figure 12 compares the compositions of the supernova ejecta as computed by adopting two different progenitor models (both having $25 \mathrm{M}_{\odot}$ ) having an amount of central $\mathrm{C}$ after the core-He burning of 0.2 and 0.4 , respectively.

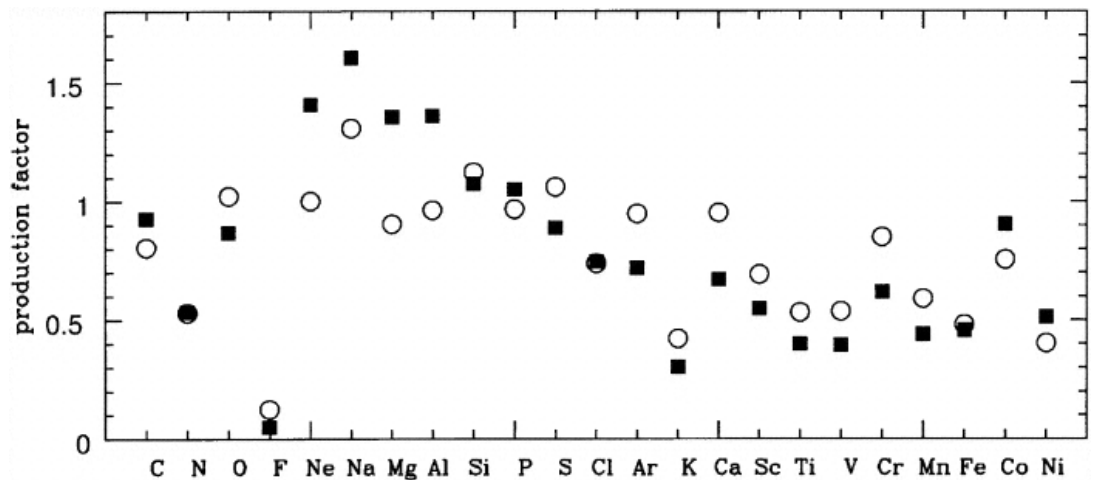

Figure 12. Production factors in the ejecta of a ore collapse supernova whose progenitor had a mass of 25 $\mathrm{M}_{\odot}$, as obtained when the $\mathrm{C}$ mass fraction left in the core by the He burning is 0.4 (filled squares) and 0.2 (open circles). Adapted from [12].

Note that intermediate-light elements, $\mathrm{Ne}, \mathrm{Na}, \mathrm{Mg}$, and $\mathrm{Al}$, which are produced in the $\mathrm{C}$ convective shell, scale directly with the $\mathrm{C}$ abundance left by the He burning (for instance, less carbon implies less magnesium). On the contrary, those elements that are produced by any of the four explosive burnings (complete explosive Si burning, incomplete explosive Si burning, explosive $\mathrm{O}$ burning, and explosive Ne burning) scale inversely with the $\mathrm{C}$. Such an occurrence depends on the smaller iron core of the low-C presupernova model, for which a more powerful explosion occurs. Finally, the iron group elements are underproduced by the low-C model, because of the smaller iron core. Note that low-C model implies yields with a nearly scaled solar distribution. This occurrence was originally pointed out by [14]. Nevertheless, because of the interplay between convection and nuclear burning and since the quantitative prescriptions for both these phenomena are still quite uncertain, we cannot use this occurrence, as often found in the extant literature, to constrain the rate of the ${ }^{12} \mathrm{C}(\alpha, \gamma){ }^{16} \mathrm{O}$ reaction. 


\section{Conclusions}

The theoretical framework described in section 2, describe a rather simplified picture of a star (spherical symmetry, hydrostatic equilibrium,, no rotation, no magnetic fields). Nevertheless it provides a powerful tool to investigate stellar physics and to interpret several astronomical observations. A quite more complex set of equations should be used for rotating stars. Actually, all stars rotate, but the majority of them (in particular low mass stars, like the Sun) are slow rotators, so that the consequences of these phenomenon can be neglected. However, when the angular momentum of a star is large enough, rotation produces sizable effects. First of all, equation (1) must be modified to account for the contribution of the centrifugal force to the hydrostatic equilibrium. This implies deviations from the spherical symmetry: the equipotential surfaces are no longer spherical, so that the entire set of equations should be rewritten in $2 \mathrm{~d}$ (at least) ${ }^{8}$. Rotation may also induce various kind of instabilities, such as meridional circulation or, in case of differential rotation, shear turbulence. When arise, these instabilities may produce important effect: they induce mixing of material as well as heat transport and angular momentum redistribution. Rotation may also imply the generation of an internal magnetic field through the dynamo mechanism. The numerical difficulty of handling multi-d stellar structures is an objective limit to the diffusion of rotating models, even if in the last decade there is a substantial increase of attention for this phenomenon.

Another important modification of the minimal set of equation here presented concerns the mass loss occurring during the evolution of a star. For the Sun, a typical main sequence star, the rate of stellar wind is $10^{-14} M_{\odot} / y r$. RGB and AGB stars suffer a definitely stronger mass loss, whose rate typically ranges between $10^{-9}$ and $10^{-5} M_{\mathcal{O}} / y r$. Owing to the difficulty in the theoretical treatment of this important phenomenon, the mass loss rate used for stellar evolution calculations is usually based on semi-empirical formulas, the most famous of one is the socalled Reimers' formula derived by [15] on the base of red giant stars observation:

$$
\dot{M}=1.354 \cdot 10^{-5} \eta \frac{L^{3 / 2}}{M T_{e f f}^{2}} \quad M_{\odot} y r
$$

where $L$ and $M$ (luminosity and mass, respectively) are in solar units, $T_{\text {eff }}$ (effective temperature) is in $\mathrm{K}$ and $\eta$ is an empirically calibrated parameter. For RGB stars $\eta$ is about $0.2-0.4$.

Further complications arises when the star belongs to a close binary system. In this case, one should carefully consider the possible interactions between the binary components that may occur during the evolution of the system. Mass and/or angular momentum transfer are quite common features of these systems. In such a way, stellar evolution may follow alternative evolutionary tracks, which cannot be accessed by single stars. Very interesting astronomical events are related to the mass transfer occurring in interactive binaries. Among them, novae, tipe Ia supernovae, X-ray binaries and cataclysm variables.

Let me finally recall that reliable stellar models may be used to move ahead the frontiers of physics and astrophysics. Several candidates for the constituents of cold dark matter has been proposed, from axions to WIMPs (weak interactive massive particles). Being massive, these particles should be sensitive to the gravitational field generated by a star, since the epoch of the

\footnotetext{
${ }^{8}$ Actualy, a 1d approximation suitable for slow rotating stars has been proposed by [1].
} 
formation. Do the physical properties and the evolution of a star change due to the influence of these particles? Can they be produced, like neutrinos, in particular conditions taking place in stellar interiors? In principle, the inclusion of the physics beyond the standard model may imply sizable modifications of the macroscopic properties of stars. If this is the case, stellar modelers and astronomers astronomers may probe new fundamental physics theories. This is not a news. The contribution of standard solar models to the comprehension of the neutrino physics is well known, indeed.

\section{References}

[1] G. R. Caughlan and W.A. Fowler, Atomic Data and Nuclear Data Tables, 1988, vol. 40, issue 2, 283-384.

[2] O. Straniero, 1988, A\&A 76. 157.

[3] R. Kippenhan, A. Weigert, 1990, Stellar Structure and Evolution, 1st edn. Springer-Verlag Berlin.

[4] E.E. Salpeter 1995, ApJ, 121, 161.

[5] O. Straniero, A. Chieffi and M. Limongi 1997, ApJ 490, 425.

[6] G. Imbriani et al., 2004, A\&A, 420, 625.

[7] B.J. Anthony-Twarog et al. 1990, AJ, 99, 1504.

[8] S. Cristallo et al. 2009, ApJ, 696, 797.

[9] P. G. Prada Moroni and O. Straniero 2002, ApJ, 581, 585.

[10] I. Dominguez, P. Hoflich and O. Straniero 2001, ApJ, 557, 279

[11] O. Straniero et al. 2003, ApJ,583, 878.

[12] G. Imbrini et al. 2001, ApJ, 558, 903.

[13] M. F. El Eid et al. 2004, ApJ, 611, 452.

[14] T.A. Weaver and S.E. Wosley 1993, Phys.Rep 227, 65.

[15] D. Reimers 1975, Mem. Soc. R. Sci. Liege, $6^{\text {e }}$ Ser. 8, 369. 